University of Nebraska - Lincoln

DigitalCommons@University of Nebraska - Lincoln

Donald Umstadter Publications

Research Papers in Physics and Astronomy

2006

Exact analytical solution for the vector electromagnetic field of Gaussian, flattened Gaussian, and annular Gaussian laser modes

Scott M. Sepke

University of Nebraska-Lincoln

Donald Umstadter

University of Nebraska - Lincoln, donald.umstadter@unl.edu

Follow this and additional works at: http://digitalcommons.unl.edu/physicsumstadter

Part of the Physics Commons

Sepke, Scott M. and Umstadter, Donald, "Exact analytical solution for the vector electromagnetic field of Gaussian, flattened Gaussian, and annular Gaussian laser modes" (2006). Donald Umstadter Publications. 98.

http://digitalcommons.unl.edu/physicsumstadter/98

This Article is brought to you for free and open access by the Research Papers in Physics and Astronomy at DigitalCommons@University of Nebraska Lincoln. It has been accepted for inclusion in Donald Umstadter Publications by an authorized administrator of DigitalCommons@University of Nebraska - Lincoln. 


\title{
Exact analytical solution for the vector electromagnetic field of Gaussian, flattened Gaussian, and annular Gaussian laser modes
}

\author{
Scott M. Sepke and Donald P. Umstadter \\ Department of Physics and Astronomy, University of Nebraska, Lincoln, Nebraska 68588-0111
}

\begin{abstract}
Received December 14, 2005; revised February 9, 2006; accepted February 10, 2006; posted February 17, 2006 (Doc. ID 66666)
The exact vector integral solution for all the electromagnetic field components of a general flattened Gaussian laser mode is derived by using the angular spectrum method. This solution includes the pure and annular Gaussian modes as special cases. The integrals are of the form of Gegenbauer's finite integral and are computed analytically for each case, yielding fields satisfying the Maxwell equations exactly in the form of quickly converging Fourier-Gegenbauer series. (C) 2006 Optical Society of America

OCIS codes: $140.3300,260.2110,350.5500$.
\end{abstract}

As laser focusing tightens to reach ever higher intensities, nonparaxial field components begin to play an important role in laser-matter interactions and must be accurately accounted for. Longitudinal laser field components were first introduced by Lax et al. ${ }^{1}$ and then discussed in relation to lasers. ${ }^{2}$ This work was quickly followed by the derivation of higher-order nonparaxial corrections for Gaussian laser beams by Davis $^{3}$ and Barton and Alexander. ${ }^{4}$ Hora and colleagues have also reviewed and contributed to the development of laser field models and their application to electrodynamics-the accuracy principle..$^{2,5-7}$ The importance of the physical model of focused laser fields has been clearly demonstrated in direct laserelectron scattering ${ }^{5,8-13}$ and must be considered to accurately model any high-intensity experiment. ${ }^{14}$

In this Letter we derive an exact analytical series solution to the full Maxwell wave equation for a laser having a flattened Gaussian transverse profile in the focal plane by using the angular spectrum method. The technique is identical to that previously used in a purely integral form for a pure Gaussian. ${ }^{5,10,15}$

Several formalisms have been developed to model flat-top laser profiles: super-Gaussians, ${ }^{16}$ weighted sums of Gaussians with different focusing parameters ${ }^{17-20}\left(1-r^{2}\right)^{1 / \gamma} \operatorname{rect}(r-1 / 2)$ and $\{1$ $+\exp [\gamma(r-1)]\}^{-1}(\gamma \in \mathbb{Z})$ mentioned by Gori, ${ }^{21}$ a super-Lorentzian, ${ }^{17}$ a wavelet technique, ${ }^{22}$ and the flattened Gaussian introduced by Gori ${ }^{21}$ and also utilized by Santarsiero and Borghi et al. ${ }^{23,24}$ All of these have been used to describe the scalar-paraxial properties of flat-top fields and are suitable in principle to generate a full vector theory for a flattened-annular focused laser beam. In this Letter, owing to the termby-term simplicity of the Fourier transform, the general flattened Gaussian distribution is chosen,

$$
E_{x}(x, y, z=0)=E_{0} \sum_{N=0}^{\infty} A_{N}\left(\frac{r^{2}}{w_{0}^{2}}\right)^{N} e^{-\left(r^{2} / w_{0}^{2}\right)},
$$

where the parameters $A_{N}$ are a set of arbitrary constants describing the detailed structure of the distribution. Note that this reduces to the Gaussian case for $A_{0}=1$ and $A_{N>0}=0$. This holds for arbitrary com- plex values of $A_{N}$, allowing a large amount of flexibility in specifying the field distribution.

When we impose the boundary condition from Eq. (1) and with $E_{y} \equiv 0$, the five remaining field components are

$$
\begin{aligned}
& E_{x}^{a}=\sum \int_{0}^{1} e^{-\left(b^{2} / \epsilon^{2}\right)} e^{i m k_{0} z} J_{0}\left(k_{0} r b\right) L_{N}\left(\frac{b^{2}}{\epsilon^{2}}\right) b \mathrm{~d} b, \\
& E_{z}^{a}=i \sum \partial_{\bar{x}} \int_{0}^{1} e^{-\left(b^{2} / \epsilon^{2}\right)} \frac{e^{i m k_{0} z}}{m} J_{0}\left(k_{0} r b\right) L_{N}\left(\frac{b^{2}}{\epsilon^{2}}\right) b \mathrm{~d} b,
\end{aligned}
$$

$$
B_{x}^{a}=\sum \partial_{x y}^{2} \int_{0}^{1} e^{-\left(b^{2} / \epsilon^{2}\right)} \frac{e^{i m k_{0} z}}{m} J_{0}\left(k_{0} r b\right) L_{N}\left(\frac{b^{2}}{\epsilon^{2}}\right) b \mathrm{~d} b,
$$

$$
\begin{aligned}
B_{y}^{a}= & \sum\left[\int_{0}^{1} e^{-\left(b^{2} / \epsilon^{2}\right)} m e^{i m k_{0} z} J_{0}\left(k_{0} r b\right) L_{N}\left(\frac{b^{2}}{\epsilon^{2}}\right) b \mathrm{~d} b\right. \\
& \left.-\partial_{\bar{x}}^{2} \int_{0}^{1} e^{-\left(b^{2} / \epsilon^{2}\right)} \frac{e^{i m k_{0} z}}{m} J_{0}\left(k_{0} r b\right) L_{N}\left(\frac{b^{2}}{\epsilon^{2}}\right) b \mathrm{~d} b\right],
\end{aligned}
$$

$$
B_{z}^{a}=i \sum \partial_{\bar{y}} \int_{0}^{1} e^{-\left(b^{2} / \epsilon^{2}\right)} e^{i m k_{0} z} J_{0}\left(k_{0} r b\right) L_{N}\left(\frac{b^{2}}{\epsilon^{2}}\right) b \mathrm{~d} b
$$

where $J_{n}(x)$ is the $n$th order Bessel function of the first kind, $L_{N}(x)$ is the $N$ th order unassociated Laguerre polynomial, $\partial_{\bar{a}}(\cdot)$ denotes $k_{0}^{-1} \partial_{a}(\cdot), \Sigma$ denotes $2 \epsilon^{-2} \widetilde{E}_{0} \Sigma_{N=0}^{\infty} A_{N} N$ !, $\widetilde{E}_{0} \equiv E_{0} \exp \left[-i\left(\omega_{0} t+\phi_{0}\right)\right]$, and $\phi_{0}$ is an arbitrary phase constant.

Imposing an identical boundary condition to $B_{y}(x, y, z=0)$ and averaging the results, the real symmetric flat-top electric field components are then

$$
E_{x}(x, y, z)=\frac{1}{2}\left(I_{1}+\frac{x^{2}-y^{2}}{k_{0} r^{3}} I_{2}+\frac{y^{2}}{r^{2}} I_{3}\right),
$$




$$
\begin{aligned}
& E_{y}(x, y, z)=\frac{1}{2} \frac{x y}{k_{0} r^{3}}\left(2 I_{2}-k_{0} r I_{3}\right), \\
& E_{z}(x, y, z)=\frac{1}{2}-I_{4} .
\end{aligned}
$$

The magnetic field is formally identical with the roles of $x$ and $y$ reversed, and the integrals $I_{n}$ are defined as

$$
\begin{aligned}
& I_{1}=\sum \int_{0}^{1} e^{-\zeta^{2}}\left(m+m^{2}\right) \sin \left(\phi_{m}\right) J_{0}(\Lambda) L_{N}\left(\zeta^{2}\right) \mathrm{d} m, \\
& I_{2}=\sum \int_{0}^{1} e^{-\zeta^{2}} \sin \left(\phi_{m}\right) J_{1}(\Lambda) L_{N}\left(\zeta^{2}\right) \sqrt{1-m^{2}} \mathrm{~d} m, \\
& I_{3}=\sum \int_{0}^{1} e^{-\zeta^{2}} \sin \left(\phi_{m}\right) J_{0}(\Lambda) L_{N}\left(\zeta^{2}\right)\left(1-m^{2}\right) \mathrm{d} m, \\
& I_{4}=\sum \int_{0}^{1} e^{-\zeta^{2}} \kappa(m) \cos \left(\phi_{m}\right) J_{1}(\Lambda) L_{N}\left(\zeta^{2}\right) \mathrm{d} m,
\end{aligned}
$$

in Eq. (10). In fact, only the case of $s=0$ needs to be computed for all $d=0,1,2, \ldots$. Once these are known, the $s=1$ terms are automatically known from the relation $a_{1, N}^{d-1}=3 a_{0, N}^{d}$ following from the properties of the Gegenbauer polynomials. Similarly, the remaining terms follow from the recursion

$$
a_{s, N}^{d}=\left(\frac{2 s+1}{s}\right)\left[a_{s-1, N}^{d+1}-\left(\frac{s-1}{2 s-3}\right) a_{s-2, N}^{d}\right],
$$

where $s=2,3,4, \ldots$ and $d=0,1,2, \ldots$ For $s=0$, the integral of Eq. (10) can be easily evaluated:

$$
\begin{aligned}
a_{0, N}^{d}= & \frac{1}{4} e^{-1 / \epsilon^{2}} \sum_{m=0}^{N} \sum_{k=0}^{m}\left(\begin{array}{l}
N \\
m
\end{array}\right)\left(\frac{1}{m !}\right)\left(\begin{array}{c}
m \\
k
\end{array}\right) \\
& \times(-i)^{\mu} \epsilon^{\mu+2 m-2 k-2} \gamma\left(\frac{2 m+\mu}{2}, \frac{1}{\epsilon^{2}}\right),
\end{aligned}
$$

where $\mu=d+1-2 k$, and $\gamma(a, x)$ is the lower incomplete gamma function. ${ }^{27}$ For a pure Gaussian (i.e., $A_{0}=1, A_{N>0}=0$ ), this can be simplified to

$$
a_{0,0}^{d}=\frac{1}{4}(-i)^{d+1} \epsilon^{d-1} e^{-1 / \epsilon^{2}} \gamma\left(\frac{d+1}{2},-\frac{1}{\epsilon^{2}}\right),
$$

as the Laguerre polynomial $L_{0}(x) \equiv 1$. Note that the inclusion of non-Gaussian terms in the boundary condition only alters the expansion coefficients and does not add any additional complexity to the problem.

The expansion coefficients of Eq. (11) are now known, and all that remains to compute the field distribution is to evaluate the derivatives of Eqs. (2)-(6), and then average this result with the analogous solution for $(x, y) \rightarrow(y, x)$. The final exact solution of the Maxwell wave equation for an arbitrarily tightly focused flattened Gaussian focal plane distribution is

$$
\begin{aligned}
E_{x}= & \sum \sum_{s=0}^{\infty} i^{s}\left\{\left(a_{s, N}^{1}+a_{s, N}^{2}\right) C_{s}^{1 / 2}\left(\begin{array}{l}
z \\
\rho
\end{array}\right) j_{s}\left(k_{0} \rho\right)-a_{s, N}^{0} \frac{x^{2}+z^{2}}{k_{0} \rho^{3}}\right. \\
& \times\left[C_{s}^{1 / 2}\left(\begin{array}{c}
z \\
\rho
\end{array}\right) j_{s}^{\prime}\left(k_{0} \rho\right)-\left(\frac{z}{k_{0} \rho^{2}}\right) C_{s}^{3 / 2}\left(\begin{array}{c}
z \\
\rho
\end{array}\right) j_{s}\left(k_{0} \rho\right)\right] \\
& +a_{s, N}^{0} \frac{y^{2}}{\rho^{2}}\left[C_{s}^{1 / 2}\left(\begin{array}{l}
z \\
-
\end{array}\right) j_{s}^{\prime \prime}\left(k_{0} \rho\right)-\frac{z}{k_{0} \rho^{2}} C_{s}^{3 / 2}\left(\begin{array}{l}
z \\
-
\end{array}\right) j_{s}^{\prime}\left(k_{0} \rho\right)\right] \\
& -a_{s, N}^{0} \frac{y^{2} z}{k_{0} \rho^{4}}\left[C_{s}^{3 / 2}\left(\begin{array}{c}
z \\
-
\end{array}\right) j_{s}^{\prime}\left(k_{0} \rho\right)\right. \\
& \left.\left.-\frac{3 z}{k_{0} \rho^{2}} C_{s}^{5 / 2}\left(\begin{array}{l}
z \\
\rho
\end{array}\right) j_{s}\left(k_{0} \rho\right)\right]\right\}
\end{aligned}
$$

where $\rho^{2}=r^{2}+z^{2}$ and $j_{s}(x)$ is the $s$ th order spherical Bessel function of the first kind. ${ }^{27}$

The problem has now been recast from evaluating the integrals of Eqs. (2)-(6) to calculating the inte- 


$$
\begin{aligned}
E_{y}= & -\left(\frac{x y}{\rho^{2}}\right) \sum \sum_{s=0}^{\infty} i^{s}\left\{a _ { s , N } ^ { 0 } \frac { 1 } { k _ { 0 } \rho } \left[C_{s}^{1 / 2}\left(\begin{array}{l}
z \\
-
\end{array}\right) j_{s}^{\prime}\left(k_{0} \rho\right)\right.\right. \\
& \left.-\left(\frac{z}{k_{0} \rho^{2}}\right) C_{s}^{3 / 2}\left(\begin{array}{l}
z \\
\frac{\rho}{\rho}
\end{array}\right) j_{s}\left(k_{0} \rho\right)\right]-a_{s, N}^{0}\left[C_{s}^{1 / 2}\left(\begin{array}{c}
z \\
\frac{\rho}{\rho}
\end{array}\right) j_{s}^{\prime \prime}\left(k_{0} \rho\right)\right. \\
& \left.-\frac{z}{k_{0} \rho^{2}} C_{s}^{3 / 2}\left(\begin{array}{c}
z \\
\frac{\rho}{\rho}
\end{array}\right) j_{s}^{\prime}\left(k_{0} \rho\right)\right] \\
& +a_{s, N}^{0} \frac{z}{k_{0} \rho^{2}}\left[C_{s}^{3 / 2}\left(\frac{z}{\rho}\right) j_{s}^{\prime}\left(k_{0} \rho\right)\right. \\
& \left.\left.-\frac{3 z}{k_{0} \rho^{2}} C_{s}^{5 / 2}\left(\begin{array}{l}
z \\
\rho
\end{array}\right) j_{s}\left(k_{0} \rho\right)\right]\right\}, \\
E_{z}= & -i\left(\frac{x}{\rho}\right) \sum \sum_{s=1}^{\infty}\left(a_{s, N}^{0}+a_{s, N}^{1}\right) i^{s}\left[C_{s}^{1 / 2}\left(\begin{array}{l}
z \\
\rho
\end{array}\right) j_{s}^{\prime}\left(k_{0} \rho\right)\right. \\
& \left.-\frac{z}{k_{0} \rho^{2}} C_{s-1}^{3 / 2}\left(\begin{array}{l}
z \\
\rho
\end{array}\right) j_{s}\left(k_{0} \rho\right)\right],
\end{aligned}
$$

for $j_{s}^{\prime}(x)=\partial_{x} j_{s}(x)=j_{s-1}(x)-[(s+1) / x] j_{s}(x), \quad$ and all Gegenbauer polynomials $C_{s}^{\lambda}$ for $s<0$ are taken by convention to be zero. The number of terms, $S$, required in the Fourier-Gegenbauer series to ensure convergence of all the field components scales roughly linearly with the waist, $w_{0}$, as $S \sim 20\left(w_{0} / \lambda_{0}\right)$, where $\lambda_{0}$ is the laser wavelength. Provided that at least this many terms are retained, the solutions are stable for all temporal and spatial locations and quickly computed relative to the integral solution. For example, the time required to compute a $77 \times 77$ field grid in the focal plane for a $2 w_{0}=\lambda_{0}$ Gaussian was decreased by a factor of 130 . As $w_{0} \gg \lambda_{0}$, the number of terms becomes large, and, in fact, the expansion coefficients become difficult to evaluate as the integrand $\exp \left(-\zeta^{2}\right)$ becomes sharply peaked in the forward direction. In this case, however, the field is now propagating nearly exactly along the $\hat{\mathbf{z}}$ axis, and the standard paraxial-perturbative solutions are applicable and, indeed, much simpler. ${ }^{40,10}$

The six symmetric electromagnetic field components of a focused laser with a general, flattened Gaussian transverse profile have been derived exactly. The formal prototype used gives the user an infinite set of fitting parameters allowing great flexibility in matching a realistic laser profile including nonideal Gaussians with flattened profiles, purposefully created flat-top beams, and even annular beams. The simplest case sets $A_{0}=1$ and $A_{N>1}=0$, reducing this to the standard Gaussian solution, and the inclusion of even one or two additional terms generates a wide array of flattened and hollow Gaussian beam profiles.
The authors acknowledge support for this work from the National Science Foundation and from the Chemical Sciences, Geosciences, and Biosciences Division of the Office of Basic Energy Sciences, U.S. Department of Energy. S. Sepke was supported in part by Sandia National Laboratories, Department of Defense-Subcontracts, award 26588. S. Sepke's e-mail address is ssepke2@unl.serve.unl.edu.

\section{References}

1. M. Lax, W. H. Louisell, and W. B. McKnight, Phys. Rev. A 11, 1365 (1975).

2. H. Hora, Physics of Laser Driven Plasmas (Wiley, 1981).

3. L. W. Davis, Phys. Rev. A 19, 1177 (1979).

4. J. P. Barton and D. R. Alexander, J. Appl. Phys. 66, 2800 (1989).

5. L. Cicchitelli, H. Hora, and R. Postle, Phys. Rev. A 41, 3727 (1990).

6. H. Hora, Laser Plasma Physics: Forces and the Nonlinearity Principle (SPIE, 2000).

7. H. Hora, M. Hoelss, W. Scheid, J. W. Wang, Y. K. Ho, F. Osman, and R. Castillo, in Proc. SPIE, 3886, 145 (2000).

8. S. Banerjee, S. Sepke, R. Shah, A. Valenzuela, A. Maksimchuk, and D. Umstadter, Phys. Rev. Lett. 95, 035004 (2005).

9. B. W. Boreham and H. Hora, Phys. Rev. Lett. 42, 776 (1979).

10. B. Quesnel and P. Mora, Phys. Rev. E 58, 3719 (1998).

11. A. Maltsev and T. Ditmire, Phys. Rev. Lett. 90, 053002 (2003).

12. H. Hora, W. Scheid, T. Hauser, Y. Kato, Y. Kitagawa, K. Mima, and T. Yamanaka, in Proceedings of the 13th International Conference on Laser Interactions and Related Plasma Phenomena, G. Miley and E. M. Campbell, eds., AIP Proc. No. 406 (AIP, 1997), p. 495.

13. S. Masuda, M. Kando, H. Kotaki, and K. Nakajima, Phys. Plasmas 12, 013102 (2005).

14. S. Weber, G. Riazuelo, P. Michel, R. Loubere, F. Walraet, V. T. Tikhonchuk, V. Malka, J. Ovadia, and G. Bonnaud, Laser Part. Beams 22, 189 (2004).

15. P. Varga and P. Török, Opt. Commun. 152, 108 (1998).

16. S. DeSilestri, P. Laporta, V. Magni, and O. Svelto, IEEE J. Quantum Electron. QE-24, 1172 (1988).

17. Y. Li, Opt. Lett. 27, 1007 (2002).

18. R. Borghi, J. Opt. Soc. Am. A 18, 1627 (2001).

19. H. Mao and D. Zhao, J. Opt. Soc. Am. A 22, 647 (2005).

20. Y. Li, Opt. Commun. 206, 225 (2002).

21. F. Gori, Opt. Commun. 107, 335 (1994).

22. R. Potvliege, J. Opt. Soc. Am. A 17, 1043 (2000).

23. M. Santarsiero and R. Borghi, J. Opt. Soc. Am. A 16, 188 (1999).

24. V. Bagini, R. Borghi, F. Gori, A. M. Pacileo, M. Santarsiero, D. Ambrosini, and G. S. Spagnolo, J. Opt. Soc. Am. A 13, 1385 (1996).

25. G. N. Watson, A Treatise on the Theory of Bessel Functions (Cambridge U. Press, 1980).

26. R. Kant, J. Mod. Opt. 40, 337 (1993).

27. M. Abramowitz and I. A. Stegun, eds., Handbook of Mathematical Functions, 12th ed. (Dover, 1972). 